\title{
Scottish national survey of tuberculosis notifications 1993 with special reference to the prevalence of HIV seropositivity
}

\author{
A G Leitch, M Rubilar, J Curnow, G Boyd, G I Forbes, S Burns, B Watt
}

Royal Victoria Chest Clinic, Chalmers Hospital, Royal Infirmary NHS Trust, Edinburgh EH3 9HQ, UK

A G Leitch

M Rubilar

Department of Public Health, Grampian Health Board

J Curnow

Glasgow Royal Infirmary

$G$ Boyd

Environmental Health (Scotland) Unit G I Forbes

Regional Virus Laboratory, City Hospital, Edinburgh S Burns

Scottish Mycobacteria Reference Laboratory, City Hospital, Edinburgh

B Watt

Correspondence to: Dr A G Leitch.

Received 1 June 1995 Returned to authors 20 August 1995 Revised version received 7 September 1995 Accepted for publication 12 September 1995

\begin{abstract}
Background - The study sought to determine the contribution of HIV seropositivity to the arrest of decline in tuberculosis notifications in Scotland. Methods - Survey forms relating to each tuberculosis notification in 1993 were completed by the notifying consultant. Voluntary anonymous HIV testing of tuberculosis cases aged under 65 was requested. Age, sex, ethnic status, country of birth, employment status, occupation, previous tuberculosis, contact status, risk factors for HIV infection, HIV serostatus of cases aged under 65, site, radiological extent, and bacteriological status of tuberculous disease were determined.
\end{abstract}

Results - Five hundred and seventy four cases of tuberculosis were originally notified, of which 77 (14\%) subsequently proved to be non-tuberculous and were therefore denotified. Of the 497 cases 423 $(85 \%)$ were white and $58(12 \%)$ were from the Indian subcontinent. Eighty five per cent of patients from the Indian subcontinent were aged $<55$ years whereas $64 \%$ of white patients were aged $>55$ years. Pulmonary disease was found in $74 \%$, nonpulmonary in $22 \%$, and combined disease in $4 \%$ of patients. Of $242 \mathrm{HIV}$ tests performed, three were positive and five other HIV positive patients were known, giving an HIV positivity rate of $1.6 \%$ of all tuberculosis notifications in 1993. Annual notification rates for Scotland were 9.7 per $10^{5}$ before and 8.7 per $10^{5}$ after exclusion of previously treated cases; rates were $8 \cdot 4$ per $10^{5}$ for the white population and 179 per $10^{5}$ for those from the Indian subcontinent. Conclusions - The study documents the distribution of tuberculous disease in Scotland by age, sex, site, and ethnic group for the first time. Notification practices, with respect to denotification, need to be improved. Infection with HIV is presently uncommon in cases of tuberculosis in Scotland but continued vigilance is essential.

(Thorax 1996;51:78-81)

Keywords: tuberculosis, Scotland, HIV, ethnic groups.

The steady fall in annual notifications for tuberculosis in Scotland stopped in 1987 and annual notification numbers remained approximately constant until 1992. ${ }^{1}$ Annual notification figures for England and Wales have followed a similar pattern which is so far unexplained. ${ }^{2}$ Other European countries such as Switzerland and Holland have recorded recent increases in tuberculosis notifications which are known to be due to disease among immigrants. ${ }^{34}$ In New York City, USA, tuberculosis notifications almost doubled in the 1980 s with most of the excess notifications occurring in HIV seropositive individuals. ${ }^{5}$ The contribution of HIV seropositivity to national tuberculosis statistics in European countries is unknown, but rates of tuberculosis in AIDS patients in Europe range from $4.7 \%$ in Italy to $46 \%$ in Portugal. ${ }^{6}$

By December 1993 there were 2055 known HIV positive individuals in Scotland (population about $5 \times 10^{6}$ ) of whom 1030 were in Edinburgh where the majority $(60 \%)$ were known to be intravenous drug users. ${ }^{7}$ Only five cases of tuberculosis in total were known to have occurred in HIV positive individuals in Edinburgh up to $1993,{ }^{8}$ but HIV serostatus has not been routinely assessed in tuberculous patients in Edinburgh or Scotland.

The present study examined the demography of all notifications for tuberculosis in Scotland in 1993 and also sought to determine the HIV serostatus of all tuberculosis patients aged under 65 years.

\section{Methods}

In Scotland notifications of tuberculosis are made on a Notification of Infectious Disease Form to the Consultant in Public Health Medicine (CPHM) with responsibility for infectious diseases in each of the 15 health board areas who make weekly returns to the Information and Statistics Division of the Common Services Agency.

In 1993 each CPHM supplied us with details of each notification received including the name of the consultant responsible for each case. The responsible consultant was sent and completed a survey form requesting information about the site, radiological extent of disease, and bacteriological status of each tuberculosis case. In addition, details of age, sex, ethnic status, country of birth, employment status, occupation, previous diagnosis or treatment for tuberculosis, contact status, and risk factors for HIV infection were sought. For tuberculosis cases under the age of 65 years a kit for voluntary anonymous blood testing for HIV status was provided which comprised an information leaflet for the patient, a permission slip to be signed by the patient, and an unlabelled blood tube which was sent to the Regional Virus Laboratory in Edinburgh with a separate form. 
Table 1 Reasons for excluding 77 cases from the original total of 574 tuberculosis notifications in 1993

\begin{tabular}{lc}
\hline Reasons for exclusion & Number \\
\hline Denotified by health board or consultant (reason & 37 \\
unstated) & \\
Proven non-tuberculous mycobacterial disease & 22 \\
Neoplastic disease & 8 \\
Contaminated bronchoscope & 5 \\
Chemoprophylaxis only & 5 \\
Total & 77 \\
\hline
\end{tabular}

This form recorded only sex, age (by age group 0-14, 15-44, 45-64 years), ethnic status, disease site, and bacteriological status, and whether the patient's HIV status was known to be positive or negative. The identity of all patients under the age of 65 who did not have an HIV test performed was concealed by Soundex coding and compared with the Soundex coded cumulative HIV register for Lothian and Scotland to check for matches. Study methods were submitted to and approved by all ethical committees in each of the 15 health board areas.

In Scotland all mycobacterial cultures are forwarded to the Scottish Mycobacteria Reference Laboratory in Edinburgh for typing and sensitivity testing. Only one positive culture in 1993 was not formally notified.

Notification rates were calculated from population figures supplied by the Information and Statistics Division of the Common Services Agency based on the 1991 population census.

\section{Results}

There were $\mathbf{5 7 4}$ notifications of tuberculosis of which 77 were excluded (table 1), leaving 497 notifications of tuberculosis which included 51 patients who had had previous treatment for tuberculosis. Twenty five cases $(5 \%)$ were detected by contact tracing, 10 of whom were aged under 15 years. Three hundred of the notified patients were either smear and culture or culture positive alone for Mycobacterium tuberculosis.

Four hundred and twenty three patients $(85 \%)$ were white, $58(12 \%)$ were from the Indian subcontinent (India, Pakistan and Bangladesh), eight were African, five Chinese, two Arabian, and one was categorised as "other". Fifty four (93\%) of those from the Indian subcontinent had been born there.

The age distribution of the white patients differed from those of the Indian subcontinent

Figure 1 Percentage of notifications in each ethnic group by age group for 423 white patients $(\square)$ and 58 from the Indian subcontinent (耳).
Table 2 Disease classification by ethnic origin

\begin{tabular}{lrlc}
\hline & White & $\begin{array}{l}\text { Indian } \\
\text { subcontinent }\end{array}$ & Other \\
\hline Pulmonary disease alone & 334 & 27 & 8 \\
Non-pulmonary disease alone & 77 & 27 & 7 \\
Combined pulmonary and & 12 & 4 & 1 \\
$\quad$ non-pulmonary disease & 12 & 58 & 16 \\
Total & 423 & &
\end{tabular}

(fig 1); $85 \%$ from the Indian subcontinent were aged under 55 years whereas $64 \%$ of white patients were 55 years or older. One hundred and fifty seven of the 423 white patients (37\%) were women compared with 27 of the $58(47 \%)$ patients from the Indian subcontinent.

Pulmonary disease alone (affecting only the lung(s)) was found in 369 (74\%) patients, nonpulmonary disease alone in $111(22 \%)$, and combined pulmonary and non-pulmonary disease in $17(4 \%)$ patients. Of those with pulmonary tuberculosis, $66(18 \%)$ had cavitating disease and $61(17 \%)$ had involvement of four or more zones of the lung. The distribution of disease varied with ethnic origin; whereas 334 $(79 \%)$ of white patients had only pulmonary disease, $77(18 \%)$ had only non-pulmonary disease and 12 (3\%) had both, while among those from the Indian subcontinent the corresponding figures were $27(47 \%), 27(47 \%)$, and four $(6 \%)$ (table 2). Details of sites of non-pulmonary disease are given in table 3 . Genitourinary disease (33\%) and pleural effusion $(17 \%)$ were more common in white patients than in those from the Indian subcontinent in whom the comparable figures were $3 \%$ and $0 \%$, respectively. Lymphatic disease was more common in patients from the Indian subcontinent $(67 \%)$ than in white patients $(26 \%)$.

Two hundred and twenty two (64\%) of the 346 white patients with pulmonary disease had positive cultures for $M$ tuberculosis of whom $167(48 \%)$ had positive smears. This compared with 20 of $31(65 \%)$ and 14 of $31(45 \%)$ of patients from the Indian subcontinent with pulmonary tuberculosis. Forty two $(55 \%)$ of the 77 white patients with non-pulmonary tuberculosis had positive cultures for $M$ tuberculosis and 15 (19\%) had positive smears compared with 9 of $27(33 \%)$ and 2 of $27(7 \%)$

Table 3 Site of disease in non-pulmonary cases*

\begin{tabular}{llll}
\hline & White & $\begin{array}{l}\text { Indian } \\
\text { subcontinent }\end{array}$ & Other \\
\hline Lymphatic & $23(26)$ & $21(67)$ & $4(50)$ \\
Genitourinary & $29(33)$ & $1(3)$ & - \\
Pleural & $15(17)$ & $-1(3)$ & - \\
Miliary & $10(11)$ & $1(3)$ \\
Bone and joint & $3(3)$ & $4(13)$ & - \\
Cold abscess & $2(2)$ & $2(6)$ & $2(25)$ \\
Gastrointestinal & $2(2)$ & $2(6)$ & - \\
Meningitis & $3(3)$ & - & - \\
Pericardium & $1(1)$ & - & - \\
Skin & $1(1)$ & - & 8 \\
Total & 89 & 31 &
\end{tabular}

Values in parentheses are percentages.

* Cases include non-pulmonary alone plus non-pulmonary associated with pulmonary disease. Three non-pulmonary patients had dual sites of disease: one white with lymphatic and gastrohad dual sites of disease: one white with lymphatic and gastrointestinal disease, one white with genitourinary disease and meningitis, and one patient from the Indian subcontinent with three patients is not tabulated. 

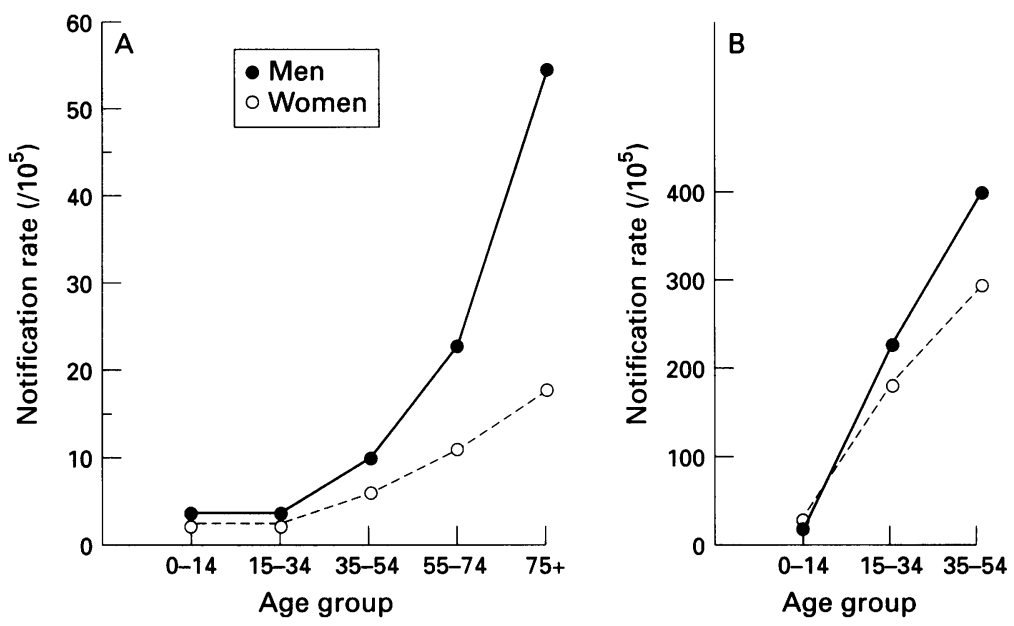

Figure 2 Tuberculosis notification rates by age and sex for $(A)$ white patients and (B) patients from the Indian subcontinent.

for those from the Indian subcontinent with non-pulmonary tuberculosis.

The most commonly recorded occupation was unskilled labourer $(18 ; 3.6 \%)$, followed by shopkeeper $(13 ; 2 \cdot 6 \%)$, shop assistant $(13$; $2 \cdot 6 \%)$, and cook $(12 ; 2 \cdot 4 \%)$. Nine shopkeepers and eight cooks were from the Indian subcontinent. Tuberculosis was notified in nine $(1.8 \%)$ schoolteachers, nine $(1.8 \%)$ nurses, and two $(0.4 \%)$ doctors. Nine men and three women were recorded as having risk factors for HIV infection. Men were at risk because of intravenous drug use (four), homosexuality or bisexuality (two), haemophilia (one), or being a sexual partner of the preceding groups (two). Three women were recorded as being sexual partners of at-risk group individuals.

Two hundred and forty two HIV tests were performed on the basis of notification for tuberculosis in individuals aged under 65 years of which three $(1 \cdot 2 \%)$ proved to be positive; one of these was previously known to be positive. One hundred and seventeen eligible patients were not HIV tested for the following principal reasons: $21(18 \%)$ refused, 18 were subsequently denotified, 17 were lost to follow up, 16 were children, 11 were known to be HIV negative, seven were due to cultural/language difficulties, six patients were deceased, and five were already known to be HIV positive. A total of eight patients from 497 tuberculosis notifications $(1.6 \%)$ were therefore known or found to be HIV positive. There were no close or identical matches between the Soundex codes of the 117 patients who qualified for, but did not receive, HIV testing and the Soundex codes for all HIV patients held on both the Lothian and Scottish HIV registers.

Of the eight HIV positive patients sex and ethnic status alone were recorded for one patient to give a total of three men and five women of whom four were white and four African. The remaining seven patients were all aged $15-44$ years; five had pulmonary and two non-pulmonary disease. One had a positive smear and culture for $M$ tuberculosis; two had negative smears and positive cultures; two had positive smears with negative cultures; and two had negative smears and cultures.
The crude notification rate based on the 1991 population census was 9.7 per $10^{5}$ in Scotland and 8.7 per $10^{5}$ after exclusion of 51 patients who had had previous treatment for tuberculosis. The notification rate for the white population in Scotland was 8.4 per $10^{5}$ with higher rates among men than women in all age groups and an increasing rate with age in both sexes (fig 2). In the Indian subcontinent population the Scottish notification rate was $179 \cdot 2$ per $10^{5}$, the rate increasing for both sexes up to 54 years of age.

\section{Discussion}

There were 574 notifications for tuberculosis in Scotland in 1993 which was a small increase on notifications in 1992, maintaining the arrest in decline or plateauing of tuberculosis notifications which began in $1987 .{ }^{1}$ Seventy seven of these notifications (14\%) were subsequently declared not to be due to tuberculosis, usually within six months of our receiving the notification, and yet most of these patients were not formally denotified. Failure to denotify is likely to have been an (unquantified) feature in previous years but, if relatively constant, suggests that the notification rate in 1993 is unchanged from previous years. Review of the Scottish Mycobacteria Reference Laboratory records of positive mycobacterial cultures identified only one patient with bone tuberculosis who had not been notified. Failure to notify bacteriologically proven cases of tuberculosis, which has been described recently in London," was therefore not a feature of the present study. The failure to denotify cases of tuberculosis identified in this study resulted in an artificial inflation by $14 \%$ of the notification figures for tuberculosis in Scotland. A two stage notification process with early notification of, for example, name, address, case record number, age, sex, site of disease, and ethnic group would ensure that contact tracing, where appropriate, is initiated. A later enquiry as to whether the notification of tuberculosis should stand and, if so, determination of the bacteriological status would complete the patient record for each individual case of tuberculosis and ensure adequate surveillance of tuberculosis nationally without the need for occasional surveys. ${ }^{1011}$

Of the patients notified with tuberculosis $5 \%$ were identified as a result of contact tracing procedures which continue to be of value in Scotland ${ }^{1213}$ and should be implemented as recently recommended by the Joint Tuberculosis Committee of the British Thoracic Society. ${ }^{14}$ A small number of cases in Scotland were also identified in members of the teaching and health professions, emphasising the need for vigilance by occupational health services. ${ }^{14}$

The ethnic status of cases of tuberculosis in Scotland has not previously been examined. Scotland has a small population from the Indian subcontinent (about 1\%) but, as in England and Wales, the incidence of tuberculosis is 20 times higher in these subjects than in the white population. ${ }^{15}$ Patients from the Indian subcontinent, as in England and Wales, ${ }^{15}$ are much younger than white patients and the pattern of 
disease is different with relatively more nonpulmonary disease than white patients and a higher incidence of lymphatic disease. Genitourinary disease was the most common nonpulmonary presentation of tuberculosis in white patients and, as in earlier studies, ${ }^{16}$ was uncommon in patients from the Indian subcontinent. Notification rates in both white subjects and those from the Indian subcontinent increased in both sexes with increasing age, the larger number of notifications of young patients from the Indian subcontinent simply reflecting the age structure of this population. The relatively different presentation of disease in patients from the Indian subcontinent should be noted by clinicians.

Voluntary anonymous blood testing for HIV serostatus in all tuberculosis notifications under the age of 65 detected two previously unidentified HIV positive patients; six other patients were already known to be HIV positive. All patients for whom serostatus was not determined had their Soundex codes compared with the Lothian and Scottish Soundex coded HIV registers. No matches were found, indicating that no known HIV positive patients had evaded testing; it remains possible that there were undiscovered HIV positive patients in this untested group. Seven of the HIV positive patients for whom the age group was given were aged 15-44 years, in keeping with the age distribution of HIV positivitity in Scotland. ${ }^{7}$ Four of the HIV positive patients were African, confirming a previous impression that tuberculosis among HIV positive overseas students might occur ${ }^{17}$ and in keeping with the considerable rise in tuberculosis notifications in England and Wales among Africans from 106 in the first six months of 1988 to 304 in the first six months of $1993 .{ }^{18}$ The overall incidence of HIV positivity detected is low ( $1.6 \%$ of all tuberculosis notifications), suggesting that at present tuberculosis is an uncommon complication of HIV infection in Scotland. We can be certain that no bacteriologically proven case of tuberculosis in Scotland in 1993 has been excluded from our analysis since all have been identified. Patients infected with HIV in Scotland are mostly young with a low incidence of previous tuberculous infection and, at present, very little evidence of reactivation of the tuberculous disease. ${ }^{8}$ Those infected with HIV are, nevertheless, at risk of new or reinfection with resultant tuberculous disease as has been well documented in North America. ${ }^{19}$ That this has not occurred yet in Scotland may reflect well on the tuberculosis service, ${ }^{20}$ but is more likely to be a function of the separation by age group of the population infected with HIV and the predominant source of infection - that is, the elderly.

This first national study of tuberculosis notifications has documented the distribution of disease by age, sex, site, and ethnic group in Scotland and confirmed the expected differences between the white population and those from the Indian subcontinent in relation to notification rates and site of disease.
Notification of bacteriologically proven cases of disease is almost complete, but denotification of cases subsequently shown to be non-tuberculous is imperfect and significantly distorts the national notification rate, suggesting the need for review of notification practices. Seropositivity for HIV infection was found in only $1.6 \%$ of cases of tuberculosis notified in Scotland in 1993 aad half of these were African.

Surveillance of tuberculosis (with improved notification practices) and HIV infection, and implementation of the guidelines for control and prevention of tuberculosis, particularly in relation to HIV infection, ${ }^{1421}$ will continue to be essential.

This study was supported by SHHD Grant No K/MRS/50/ C1838. We are indebted to our medical and surgical colleagues throughout Scotland for their full cooperation in the Study. Dr David Goldberg, Ruchill Hospital and (the late) Dr George Bath, Lothian Health, generously linked study data with Scottish Bath, Lothian Health, generously linked study data with Scottish and Lothian HIV registers. We are grateful to Mr A Rayner and Mrs G Harris of the Scottish Mycobacteria Reference Laboratory for expert technical assistance and to Miss J Holywell
as the research assistant responsible not only for the efficient functioning of the survey but also for typing this paper.

1 Scottish Office. Home and Health Department. 1993 Health in Scotland. Edinburgh: HMSO.

2 Watson JM. Tuberculosis in Britain today. BMf 1994;306 220-1.

3 Zellweger JP, Raeber PA, Desgrandchamp D. Screening for tuberculosis among immigrants entering Switzerland. Tuberc Lung Dis 1994;75(Suppl 1):44

4 Keizer ST, Année JACM, Deutekom H van, Warris-Versteegen AA. Screening for tuberculosis among immigrants and asylum seekers in the Netherlands: methods and results. Tuberc Lung Dis 1994;75(Suppl 1):30.

5 Brudney K, Dobkin J. Resurgent tuberculosis in New York City. Human immunodeficiency virus, homelessness, and the decline of tuberculosis control programs. Am Rev Respir Dis 1991;444:745-9.

6 Schwoebel V. Epidemiological data on HIV-associated tuberculosis in Europe. 2nd European Workshop on Tuberculosis Control in Low Prevalence Countries, Wolfheze, The culosis Control in Low Prevalence

7 Answer. AIDS News Supplement. CDS Weekly Report 1993; CDS 93/05.

8 Leitch AG, Rubilaw M, Watt B, Laing R, Brettle RP, Leen CLS. Disease due to $M$ tuberculosis is less common than expected in HIV-positive patients in Edinburgh. Tuberc Lung Dis 1994;75(Suppl 1):4.

9 Sheldon CD, King K, Cock H, Wilkinson P, Barnes NC. Notification of tuberculosis: how many cases are never reported? Thorax 1992;47:1015-8.

10 Rieder HL. Misbehaviour of a dying epidemic: a call for less speculation and better surveillance. Tuberc Lung Dis 1992;73:181-3

11 WHO/IUATLD Working Group on Standardised Tuberculosis Surveillance in Europe. 2nd European Workshop on Tuberculosis Control in Low Prevalence Countries, Wolfheze, The Netherlands, 20 March 1994.

12 Capewell S, Leitch AG. The value of contact procedures for tuberculosis in Edinburgh. $B r \mathcal{F}$ Dis Chest 1984;78: for tubercu

13 Rubilar M, Brochwicz-Lewinski M, Anderson M, Leitch AG. The outcome of contact procedures for tuberculosis in Edinburgh, Scotland 1982-1991. Respir Med 1995;89: 113-20.

14 Joint Tuberculosis Committee of the British Thoracic Society. Control and prevention of tuberculosis in the United Kingdom: Code of Practice 1994. Thorax 1994;49:1193200.

15 Medical Research Council Cardiothoracic Epidemiology Group. National survey of notifications of tuberculosis in England and Wales in 1988. Thorax 1992;47:770-5.

16 Moudgil H, Leitch AG. Extrapulmonary tuberculosis in Lothian 1980-1989; ethnic status and delay from onset of symptoms to diagnosis. Respir Med 1994;88:507-10.

17 Faccenda J, Watt B, Leitch AG. Increase of tuberculosis in overseas students of higher education in Edinburgh in 1991; a taste of things to come? Respir Med 1994;88: 1991; a

18 Anonymous. Tuberculosis in 1993: preliminary results of the national survey. Comm Dis Rep 1994;4:235.

19 Daley CL, Small PM, Schecter GT, et al. An outbreak of tuberculosis with accelerated progression among persons infected with the human immunodeficiency virus. An infected with the human immunodeficiency virus. An analysis using restriction-fragment-len

20 Leitch AG. Setting up and running a local tuberculosis service. Br $\mathcal{F}$ Dis Chest 1987;81:6-13.

21 Sub-Committee of the Joint Tuberculosis Committee of the British Thoracic Society. Guidelines on the management of tuberculosis and HIV infection in the UK. BMF 1992; 304:1231-3. 